\title{
A finite element simulation of transition-edge sensor for measuring kinetic energy of fission fragments
}

\author{
Xianglei Wang ${ }^{1, *}$ \\ ${ }^{1}$ Key Laboratory of Particle \& Radiation Imaging (Tsinghua University), Ministry of Education, Beijing, 100084, China.
}

\begin{abstract}
It is necessary to accurately measure the kinetic energy of fission fragments when using the Time-Of-Flight method to determine the mass of fission fragments. The ionization chamber and the Au-Si surface barrier detector are conventional kinetic-energy detectors, but their energy resolution is not sufficient to achieve a mass resolution of $1 \mathrm{amu}$. The Transition-Edge Sensor (TES) is a cryogenic calorimeter that can be used to measure the kinetic energy by measuring the temperature variation induced by the energy of the incident particle, with a typical resolution of $0.02 \%$ of TES detector can be achieved ${ }^{[1]}$. In this article, we designed a TES to measure the kinetic energy of fission fragments, and the signals of this TES with different incident particle positions, kinetic energy, and thermal conductivity were simulated using ANSYS. Therefore, we verified the feasibility of the TES and improved the count rate of the TES to $100 \mathrm{cps}$.
\end{abstract}

\section{Introduction}

The fission yield data of neutron-induced nuclear fission is of considerable significance in quite a few aspects such as nuclear structure theory and the nuclear energy industry. At present, the yield data of neutron-induced fission in those nuclear databases has pretty high uncertainty, even conflict with each other in several energy regions. Hence, much attention has been paid to the yield data, and several projects such as VAMOS ${ }^{[2]}$, SOFIA, SPIDER ${ }^{[3]}$, VERDI ${ }^{[4]}$, and CNDC have been implemented to measure the fission yield data. Also, the goal of our project is to measure the mass yield data more accurately.

The commonly used method for mass measurement of fission fragments is the TOF method. The TOF method needs to measure the kinetic energy of the primary fission product accurately. Ionization chambers and Au-Si surface barrier detectors are conventional kinetic-energy detectors, but the energy loss of the entrance window of the ionization chamber is difficult to calibrate, and the signal amplitude reduction of $\mathrm{Au}-\mathrm{Si}$ surface barrier detectors is severe. Consequently, they cannot meet the requirement of the TOF method. The TES calorimeter is an ultra-high energy resolution detector with increasing attention in recent decades. Its energy resolution is an order of magnitude higher than semiconductor detectors. In 2006-2012, several TES calorimeters were made by NIST and LOSA to measure alpha particles, whose energy resolutions are on the order of $0.02 \%-0.05 \%{ }^{[5]}$.

When a superconducting film is at its critical temperature, its resistance changes rapidly with temperature ${ }^{[6]}$. Therefore, the superconducting film can become an extremely sensitive thermometer. The main components of the TES calorimeter (see Fig. 1) are an absorber, a superconducting film, a SQUID current preamplifier, a voltage amplifier, a $\mathrm{Si}_{3} \mathrm{~N}_{4}$ film, and a $\mathrm{Si}$ substrate. A TES calorimeter measures the kinetic energy of incident particles through using a superconducting film to measure the temperature pulse caused by the kinetic energy. First, particles enter the absorber and transfer their kinetic energy to the absorber. Then the superconducting film is heated by the absorber, and generate a temperature pulse. Afterwards, under the effect of a bias voltage, the temperature pulse is converted into a current pulse and sent to the SQUID. Finally, the SQUID will amplify the current pulse, convert it into a voltage pulse, and then output it to a voltage amplifier to form signals. The heat flows into the Si substrate by the $\mathrm{Si}_{3} \mathrm{~N}_{4}$ film.

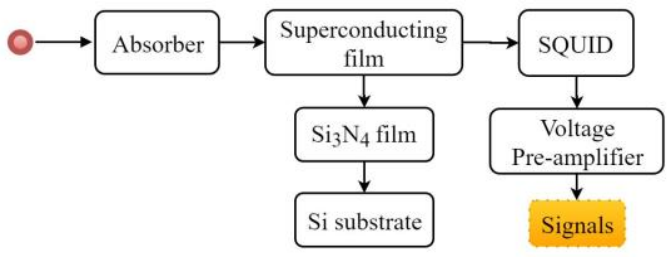

Fig. 1. The main components of the TES. Also, the measurement process is shown.

In section 2 and 3, we designed and modelled a TES calorimeter with a range slightly over $100 \mathrm{MeV}$ and a count rate around 10-100 cps. In section 4, we simulated the TES calorimeter to verify its feasibility and improve it by ANSYS (a finite element simulation software).

\footnotetext{
* Corresponding author: wxl17@mails.tsinghua.edu.cn
} 


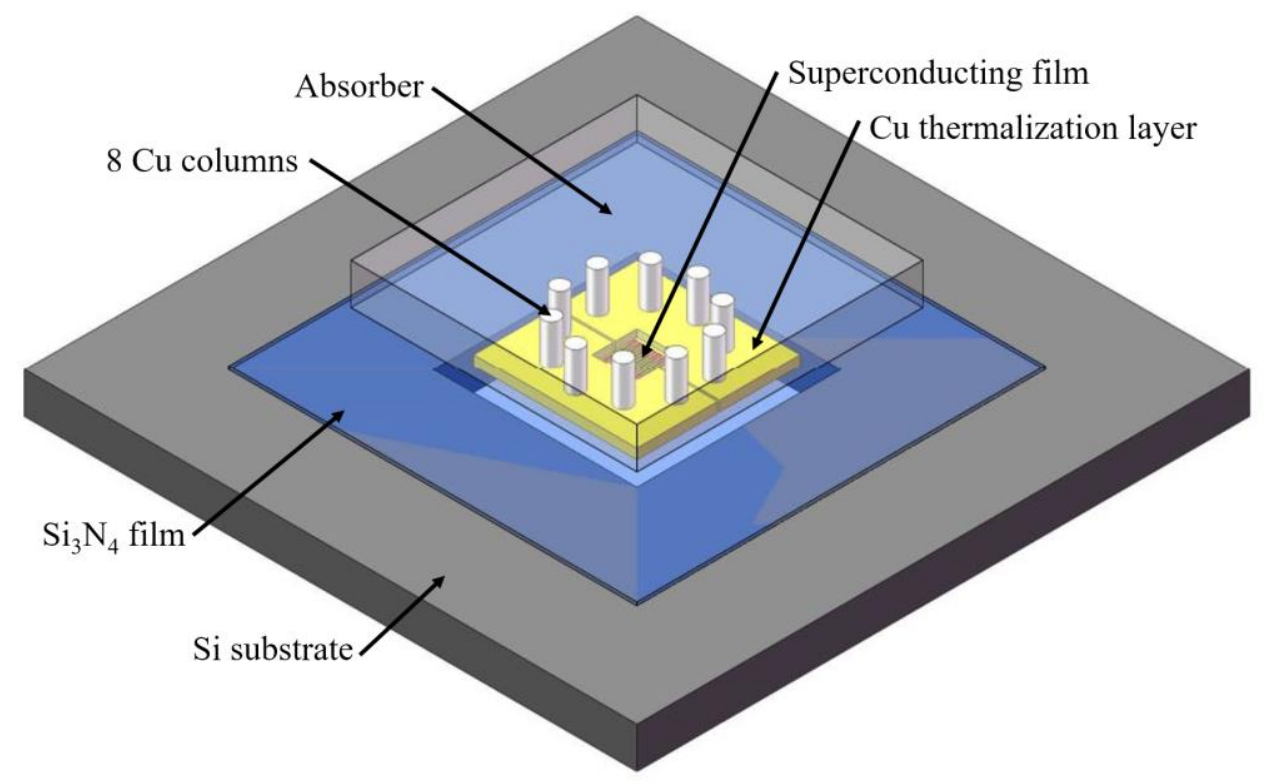

Fig. 2. A schematic drawing of the TES of our design (not to scale).

Table 1. The geometric parameters of the TES design.

\begin{tabular}{cccccc}
\hline & Absorber & $\begin{array}{c}8 \mathrm{Cu} \\
\text { columns }\end{array}$ & $\begin{array}{c}\text { Thermalization } \\
\text { layer }\end{array}$ & $\begin{array}{c}\text { Superconducting } \\
\text { film }\end{array}$ & $\mathrm{Si}_{3} \mathrm{~N}_{4}$ film \\
\hline $\begin{array}{c}\text { Cross area } \\
\left(\mu m^{2}\right)\end{array}$ & $6.4 \times 10^{7}$ & 1963.5 & $3.6 \times 10^{7}$ & $1.6 \times 10^{5}$ & $1 \times 10^{8}$ \\
$\begin{array}{c}\text { Height } \\
(\mu \mathrm{m})\end{array}$ & 12 & 10 & 1 & 0.2 & 1.5 \\
\hline
\end{tabular}

\section{Design of the TES calorimeter}

Fig. 2 shows the structural schematic of the TES of our design, while its geometric parameters are shown in Table 1. The TES calorimeter consists of an Au absorber, $8 \mathrm{Cu}$ support columns, a $\mathrm{Cu}$ thermalization layer, a $\mathrm{Ti} / \mathrm{Au}$ superconducting film, a $\mathrm{Si}_{3} \mathrm{~N}_{4}$ film, and a $\mathrm{Si}$ substrate. The $\mathrm{Au}$ absorber, the $\mathrm{Cu}$ support columns, the $\mathrm{Cu}$ thermalization layer, and the superconducting film are suspended in the centre of the Si substrate through the $\mathrm{Si}_{3} \mathrm{~N}_{4}$ film. Although the bias circuit is not printed in Fig. 1, there are two tunnels left for the bias circuit in the $\mathrm{Cu}$ thermalization layer.

It is well known that the maximum measurable energy of the calorimeter depends on the total heat capacity $\mathrm{C}$ and the transition temperature width $\delta \mathrm{T}$. The volume of the superconducting film is negligible, then the total heat capacity of the detector is dependent on the $\mathrm{Au}$ absorber, the $\mathrm{Cu}$ columns, and the $\mathrm{Cu}$ thermalization layer,

$$
C_{A}=C_{A u}+C_{C u, c o l}+C_{C u, t h} \approx 5.5 \mathrm{~nJ} / \mathrm{K}
$$

Where $C_{A}$ is the total heat capacity, $C_{A u}$ is the heat capacity of the absorber, $C_{C u, c o l}$ is the heat capacity of the $\mathrm{Cu}$ columns, $C_{C u, t h}$ is the heat capacity of the $\mathrm{Cu}$ thermalization layer. When a $100 \mathrm{MeV}$ incident particle shoots in Au absorber, the temperature pulse amplitude is around $3 \mathrm{mK}$, which approximately equals the transition temperature width of the superconducting film.
Robert D. Horansky et al. built a TES calorimeter to measure $5 \mathrm{MeV}$ particles, whose total heat capacity is about $250 \mathrm{pJ} / \mathrm{K}$, and temperature pulse amplitude is 3.2 $\mathrm{mK}^{[1]}$. Consequently, $3 \mathrm{mK}$ is acceptable for the design of TES.

The kinetic energy of the incident particles is finally converted into thermal energy and flows into the $\mathrm{Si}$ substrate through the $\mathrm{Si}_{3} \mathrm{~N}_{4}$ film, and then the detector returns to its initial state afterwards. The $\mathrm{Si}_{3} \mathrm{~N}_{4}$ film and the $\mathrm{Si}$ substrate constitute the heat dissipation structure of the TES calorimeter together. Therefore, the intrinsic heat recovery time constant $\tau_{\text {In }}$ of the TES calorimeter is

$$
\tau_{\text {In }}=\frac{C_{A}}{G}
$$

Where $G$ is the thermal conductance between the TES and the $\mathrm{Si}$ substrate. The $\mathrm{Cu}$ thermalization layer greatly expands the length of the heat dissipation boundary and obtains a high thermal conductance of almost $30 \mathrm{nW} / \mathrm{K}$. Thus, the intrinsic heat recovery time constant is roughly $180 \mathrm{~ms}$. Thereafter, considering the electro-thermal negative feedback effect through the Eq. 3 .

$$
\tau_{1}=\frac{\tau_{\text {In }}}{1+L}
$$

Where $\tau_{1}$ is the real heat recovery time constant, $L$ is the low-frequency gain factor whose typical value is 5 . Therefore, $\tau_{1}$ is around $30 \mathrm{~ms}$, and then the count rate of the TES is almost $10 \mathrm{cps}$.

\footnotetext{
Corresponding author: wxl17@mails.tsinghua.edu.cn
} 


\section{Modeling of the TES calorimeter}

As shown in Table 1, those components of TES have incredibly high aspect ratios up to 10000 , which makes it challenging to model and mesh for finite element simulation. To simplify the modeling and meshing of the TES calorimeter, the TES was divided into Model A and Model B according to the simulation time scale.

Model A, as shown in Fig. 3, includes the absorber, 8 $\mathrm{Cu}$ support columns, the thermalization layer, and the superconducting film. The simulation time scale of Model A is on the order of microseconds. According to the work of IJ Maasilta et al. ${ }^{[7]}$, we knew that the heat dissipation power from the TES to the $\mathrm{Si}$ substrate is roughly $30 \mathrm{nW} / \mathrm{K}$. Therefore, the heat dissipation in the simulation process is on the order of fJ, much smaller than $100 \mathrm{MeV}$. Hence, the $\mathrm{Si}_{3} \mathrm{~N}_{4}$ film and the $\mathrm{Si}$ substrate were neglected reasonably.

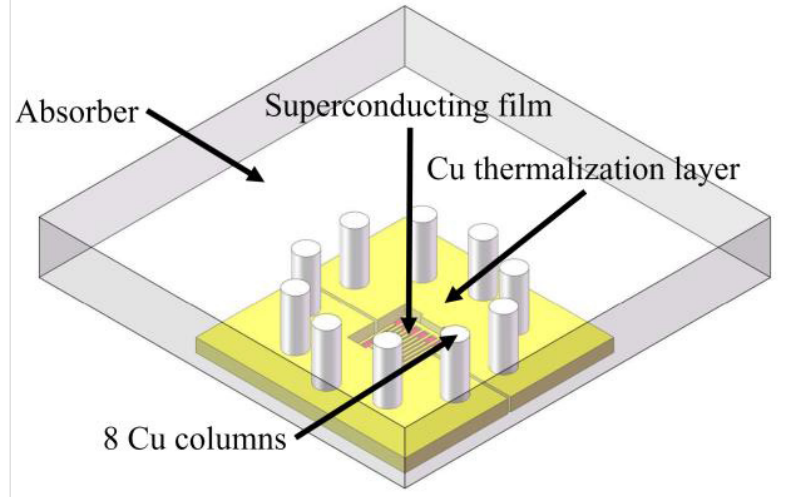

Fig. 3. The structural diagram of Model A. Removed the Si substrate and the $\mathrm{Si}_{3} \mathrm{~N}_{4}$ film compared to complete TES.

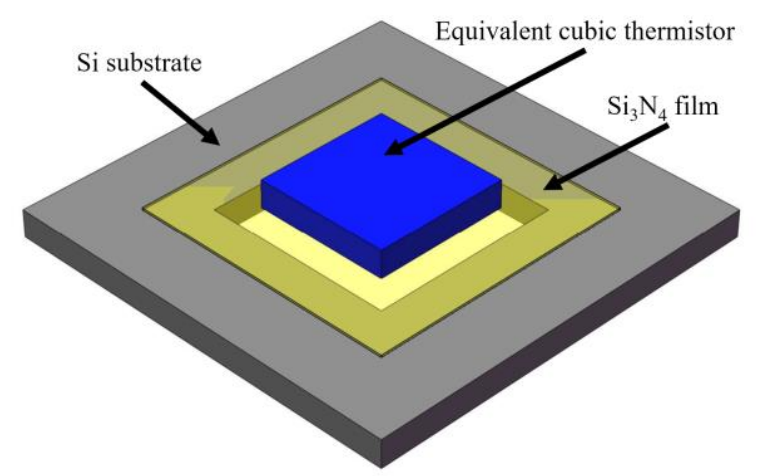

Fig. 4. The structure diagram of Model B. Replaced the absorber, the superconducting film, and the thermalization layer with a cubic thermistor.

Unlike Model A, the simulation time scale of Model $\mathrm{B}$ is on the order of milliseconds. As shown in section 4.1.1, the thermal equilibrium time constant $\tau_{2}$ between the absorber and the superconducting film is on the order of 10 microseconds, much smaller than milliseconds. Therefore, we supposed that the absorber, the $\mathrm{Cu}$ support columns, the thermalization layer, and the superconducting film have the same temperature when we simulated Model B. And then, we equated them with a voltage-biased cubic thermistor. The cubic thermistor has the same resistance with the superconducting film, the same heat capacity with $C_{A}$, and the same heat dissipation boundary length with the $\mathrm{Cu}$ thermalization layer. By this way, we obtain a simple Model B (see Fig. 4), which merely contains a cubic thermistor, a $\mathrm{Si}_{3} \mathrm{~N}_{4}$ film, and a Si substrate.

In a finite element simulation mesh, to ensure the accuracy of the simulation, one direction must be divided into at least three parts. Considering the accuracy and the limitation of computing ability, we divided the model into $2500 \mu \mathrm{m}^{2} \times 0.25 \mu \mathrm{m}$ bricks. Consequently, the max aspect ratio of the mesh is 200 .

\section{Simulated results and discussion}

After designing, modeling and meshing the TES calorimeter. In section 4.1, we used Model A to simulate the thermal coupling between the absorber and the superconducting film. Moreover, in section 4.2, we used Model $\mathrm{B}$ to simulate the current signal of the TES detector and its influencing factors.

\subsection{The thermal coupling between the absorber and the superconducting film}

In section 4.1.1, we focused on the thermal conductance $G_{1}$, where $G_{1}$ is the thermal conductance between the absorber and the $\mathrm{Cu}$ thermalization layer. If the size of $G_{1}$ is close to or even bigger than $G$, the heat leakage will become severe, and the signal will be significantly reduced. In section 4.1.2, we investigated the degree of dependence between the thermal equilibrium of the components and the location of the incident particles. If the thermal equilibrium condition is highly dependent on the incident position of the particles, the energy resolution will degrade.

\subsubsection{Thermal conductance $\mathrm{G}_{1}$}

To simulate $G_{1}$, we set the temperature of the superconducting film to $0.12 \mathrm{~K}$ and applied a varied heat power $P_{I}$ to the absorber. The simulated result about $P_{1}$ and the temperature of the absorber $T$ is shown in Table 2 . Then we obtained a $G_{1} \approx 2 \times 10^{-6} \mathrm{~W} / \mathrm{K}$ by fitting $P_{l}$ and $T$ with a linear function.

Table 2. The simulated result about $\mathrm{P}_{1}, \mathrm{~T}$, and $\Delta \mathrm{T}=\mathrm{T}-0.12$. $G_{1} \approx 2 \times 10^{-6} \mathrm{~W} / \mathrm{K}$ was fitted by those data.

\begin{tabular}{ccccccc}
\hline & \multicolumn{6}{c}{$\mathrm{P}_{1}\left(\times 10^{-8} W\right)$} \\
\cline { 2 - 7 } & 300 & 150 & 30 & 15 & 3 & 1.5 \\
\hline $\mathrm{T}(\mathrm{K})$ & 2.10 & 1.11 & 0.32 & 0.22 & 0.14 & .0 .13 \\
$\Delta \mathrm{T}(\mathrm{K})$ & 1.98 & 0.99 & 0.2 & 0.1 & 0.02 & 0.01 \\
\hline
\end{tabular}

As mentioned above, $G$ is about $30 \mathrm{nW} / \mathrm{K}$, which is much larger than $G_{1}$. Therefore, the heat leaking to the $\mathrm{Si}$ substrate can be neglected. The thermal equilibrium time constant between the absorber and the superconducting film is 


$$
\tau_{2}=\frac{C}{G_{1}}
$$

Where $C=\frac{C_{A} \times C_{\text {sup }}}{C_{A}+C_{\text {sup }}}$ is the equivalent heat capacity, $C_{A}$ is the total heat capacity calculated above, and $C_{\text {sup }}$ is the heat capacity of the superconducting film. In section 4.1.2, we simulated $\tau_{2}$ by transient simulation and compared the simulated value with predicted $9.41 \mu$ s.

\subsubsection{The influence of particle's incident position}

We simulated how the particle's incident position influences the thermal equilibrium process of Model A. In our simulation, the incident particle was equivalent to a $0.25 \mathrm{~mm}^{2}$ heat source, whose total heat equalled the incident particle's kinetic energy, and whose duration was $220 \mathrm{~ns}$ according to Kent D Irwin et al. ${ }^{[8]}$. Five 0.25 $\mathrm{mm}^{2}$ squares (see Fig. 5) in the absorber were selected to be heat sources and simulated, respectively. Then the simulated thermal equilibrium time constants and the predicted one are shown in Table 3.

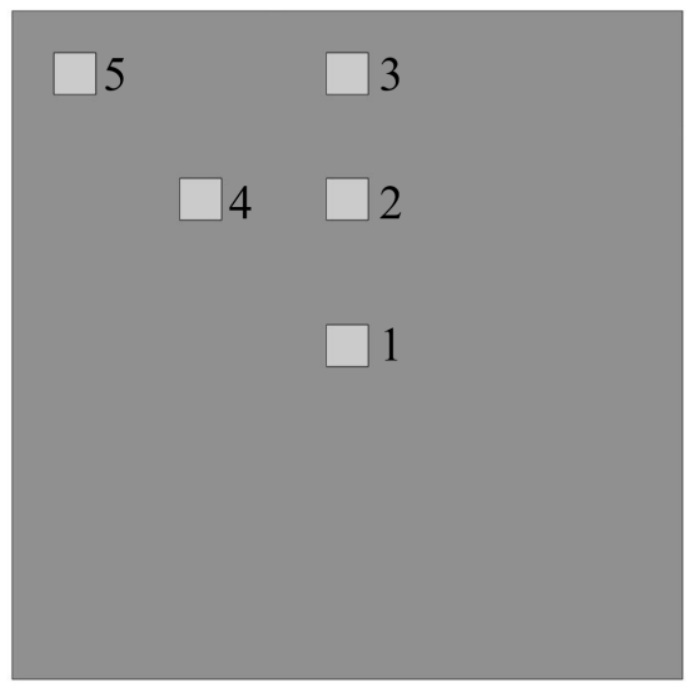

Fig. 5. Five squares selected to be equivalent heat sources and numbered by $1-5$

. Table 3. Thermal equilibrium time constants simulated with different incident locations of particles and calculated.

\begin{tabular}{ccccccc}
\hline position & 1 & 2 & 3 & 4 & 5 & Predicted \\
\hline$\tau_{2}(\mu \mathrm{s})$ & 2.5 & 10 & 10.16 & 10 & 10.2 & 9.41 \\
\hline
\end{tabular}

As shown in Table 3, when a particle enters from the center of the absorber, the time constant is $2.5 \mu \mathrm{s}$. However, it rises to around $10 \mu$ s abruptly when the incident location is $1.75 \mathrm{~mm}$ away from the centre. Then it increases slowly with the distance between the incident location and the centre. At most locations, their time constants are all around $10 \mu \mathrm{s}$, which is close to the calculated $9.41 \mu \mathrm{s}$. Therefore, $\tau_{2}$ is significantly smaller than $\tau_{1}$, the difference caused by the incident location of the particle is negligible.

\subsection{Two influence factors of the TES signals}

There are two influence factors of TES signals simulated and discussed by Model B. One is the energy of incident particles; the other one is the thermal conductance $G$ between the TES and the Si substrate.

\subsubsection{The energy of incident particles}

In our design, the expected maximum measurable incident particle energy is slightly above $100 \mathrm{MeV}$. Thus, we simulated and discussed the effects of incident particle energy on signal amplitude and signal duration. In the simulation, the cubic thermistor had a bias voltage of $4.876 \times 10^{-6} \mathrm{~V}$, a temperature of $0.12 \mathrm{~K}$, a resistance of $0.02 \Omega$, and a heat pulse whose duration is $220 \mathrm{~ns}$. Moreover, the total heat of the heat pulse changed with the energy of the incident particle. The simulated result is shown in Fig. 6.

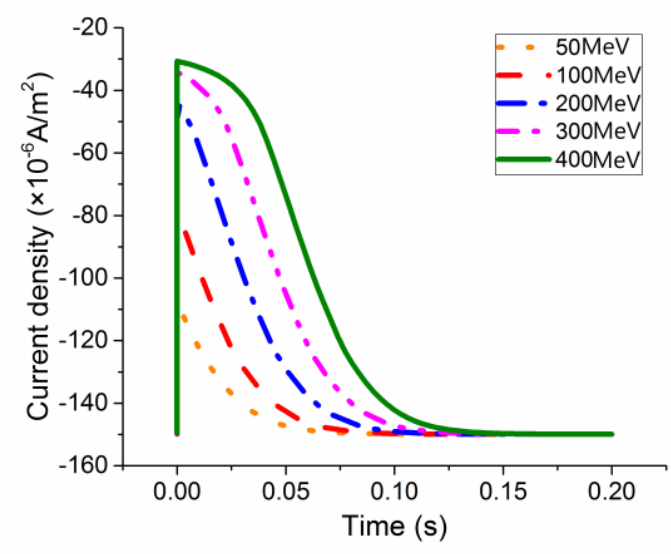

Fig. 6. TES detector signals with different energy of incident particles. As shown, the signals with energy below or close to $100 \mathrm{MeV}$, is exponentially reduced as expected. However, the signals with energy above $100 \mathrm{MeV}$, whose peaks are saturated.

As shown in Figure 6, when the incident particle energy is below $100 \mathrm{MeV}$, the signal is exponentially attenuated, and its amplitude is proportional to the incident particle's energy, which proves that our TES design can adapt $100 \mathrm{MeV}$ particle measurement. For 50 $\mathrm{MeV}$ and $100 \mathrm{MeV}$ curves, the time constants are about $20 \mathrm{~ms}$, which is close to $30 \mathrm{~ms}$ predicted in section 2 . It is worth noting that when the incident particle energy is too large (e.g. above $100 \mathrm{MeV}$ ), the TES will be saturated. Therefore, the signal amplitude will no longer be proportional to the particle energy, and the signal duration will become longer.

\subsubsection{Thermal conductance $G$}

The thermal conductivity $G$ between the TES and the Si substrate is an important design parameter for the TES calorimeter. It significantly affects the count rate and the energy resolution. To discuss the effect of $G$, we simulated the current signal when $G$ changed from 100 $\mathrm{nW} / \mathrm{K}$ to $400 \mathrm{nW} / \mathrm{K}$, and the incident particle energy was $50 \mathrm{MeV}$. Figure. 7 shows the simulated results. 


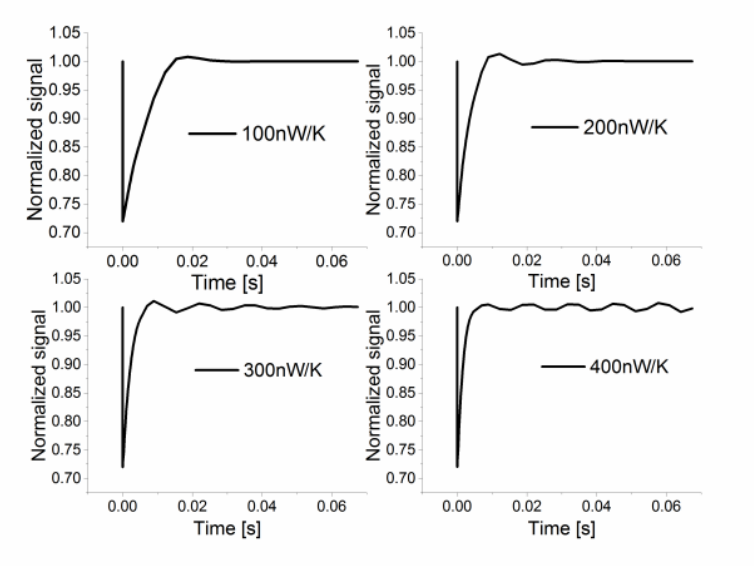

Fig. 7. Normalized TES current signals with different thermal conductance $G .100 \mathrm{nW} / \mathrm{K}$ (left-top), $200 \mathrm{nW} / \mathrm{K}$ (right-top), $300 \mathrm{nW} / \mathrm{K}$ (left-bottom), $400 \mathrm{nW} / \mathrm{K}$ (right-bottom).

As shown in Figure 7, as the thermal conductivity increases, the signal duration rapidly decreases as we expected. However, no significant pulse amplitude reduction is observed, this is because although $G$ has increased to $400 \mathrm{nW} / \mathrm{K}$, it is still much smaller than $G_{1}=$ $2 \times 10^{-6} \mathrm{~W} / \mathrm{K}$. However, when the thermal conductivity is bigger than $100 \mathrm{nW} / \mathrm{K}$, an oscillation arises at the tail of the signal, and gradually becomes stronger as the thermal conductivity increases. As a result, the signal duration of the TES is prolonged. When the thermal conductivity reaches $400 \mathrm{nW} / \mathrm{K}$, the oscillation will not stop. This oscillation may be induced by the too strong electro-thermal negative feedback effect of the superconducting film due to the excessive thermal conductivity.

In conclusion, based on the simulated results, we can increase the thermal conductivity to around $200 \mathrm{nW} / \mathrm{K}$, and then obtain a count rate that is almost $100 \mathrm{cps}$.

\section{Conclusions}

In this article, we used finite element simulation to verify and reform our TES design. As a result, we negated the influence of incident position on the detector signal, demonstrated the feasibility of the TES calorimeter, and improved the counting rate from $10 \mathrm{cps}$ to $100 \mathrm{cps}$. This article shows us how helpful the finite element simulation can be in the design process of a TES detector. We will use finite element analysis tools deeper.

There is another helpful TES model, which was called "two-fluid model." By the use of this "two-fluid model," we can make use of fully developed circuit design tools like LTSpice to simulate TES and get more meaningful results, this is what we are going to do.

\section{References}

1 Robert D Horansky, Joel N Ullom, James A Beall, Gene C Hilton, Kent D Irwin, Donald E Dry, Elizabeth P Hastings, Stephen P Lamont,
Clifford R Rudy, and Michael W \%J Applied Physics Letters Rabin, 93 (12), 123504 (2008). M Caamano, O Delaune, F Farget, X Derkx, KH Schmidt, L Audouin, C-O Bacri, G Barreau, J Benlliure, and E \% J Physical Review C Casarejos, 88 (2), 024605 (2013).

K Meierbachtol, F Tovesson, D Shields, Charles Arnold, R Blakeley, T Bredeweg, M Devlin, AA Hecht, LE Heffern, J \%J Nuclear Instruments Jorgenson, Spectrometers Methods in Physics Research Section A: Accelerators, Detectors, and Associated Equipment, 788, 59 (2015).

MO Frégeau and S \% J Physics Procedia Oberstedt, 64, 197 (2015).

M. P. Croce, M. K. Bacrania, E. M. Bond, D. E. Dry, A. L. Klingensmith, W. A. Moody, S. P. LaMont, M. W. Rabin, J. H. Rim, J. A. Beall, D. A. Bennett, V. Kotsubo, R. D. Horansky, G. C. Hilton, D. Schmidt, J. N. Ullom, and R. Cantor, IEEE Transactions on Applied Superconductivity 21 (3), 207 (2011).

$6 \quad$ Kent D Irwin and Gene C Hilton, in Cryogenic particle detection (Springer, 2005), pp. 63.

IJ Maasilta and $\mathrm{T} \% \mathrm{~J}$ Journal of Low Temperature Physics Kühn, 151 (1-2), 64 (2008).

8 Kent D Irwin, Gene C Hilton, David A Wollman, and John M \% Journal of Applied Physics Martinis, 83 (8), 3978 (1998). 